\title{
Neoadjuvante und adjuvante Therapiekonzepte beim Kolorektalkarzinom
} Neoadjuvant and Adjuvant Treatment Concepts in Colorectal Cancer

\author{
H.J. Schmoll \\ Klinik und Poliklinik für Innere Medizin, Martin-Luther-Universität Halle
}

D ie adjuvante Chemotherapie gilt international als Standard im Stadium III des kolorektalen Karzinoms und mit E inschränkungen auch bei H ochrisikopatienten des Stadiums II. $\mathrm{R}$ andomisierte Studien haben belegt, dass durch eine adjuvante 5-Fluorouracil-Therapie (in K ombination mit L evamisol oder Folinsäure) die M ortalität im Stadium III nach 3- 5J ahren um etwa ein D rittel gesenkt wird. D ie klinische Forschung ist allerdings bestrebt, die R ezidivrate weiter zu verringern. So gibt es $\mathrm{H}$ inweise aus einer Metaanalyse, dass mit höherer 5-Fluorouracil-D osis bzw. mit der D osisintensität in den ersten 3 M onaten der Therapie das Mortalitätsrisko abnimmt [1]. $D$ ies wiederum zeigt, dass eine Wirksamkeitssteigerung der adjuvanten Chemotherapie lohnend und sinnvoll ist.

\section{Neue Ansätze in der adjuvanten Therapie}

In jüngster Zeit sind daher eine R eihe randomisierter Studien angelaufen, in denen verschiedene neue Therapieansätze untersucht werden. So wird beispielsweise ein 5-FluorouracilInfusionsschema mit der konventionellen 5-FluorouracilBolustherapie verglichen, aber auch neue Substanzen wie I rinotecan oder O xaliplatin werden geprüft, teilweise in Kombination mit biologischen Wirkprinzipien wie A ntikörpern und antiangiogenen Faktoren. D ie E rgebnisse dieser Studien werden allerdings erst in frühestens 4-5 Jahren vorliegen. Wenn man 5-Fluorouracil/Folinsäure (5-FU/FA) als Bolus gegeben an seiner Wirksamkeit beim metastasierten kolorektalen $\mathrm{K}$ arzinom misst, wo die A nsprechraten im Bereich von 10-20\% liegen, so sind von einem 5-FU/FA -Infusionsschema mit einer Remissionsrate über $20-30 \%$ in der adjuvanten Situation nur marginale Verbesserungen des Therapieergebnisses zu erwarten. V ielversprechender erscheint da die Kombinationstherapie von 5-FU/FA mit I rinotecan oder Oxaliplatin, mit der in der First-line-Therapie beim metastasierten kolorektalen $\mathrm{K}$ arzinom objektive R emissionsraten von etwa $50 \%$ erzielt werden konnten. Ü berträgt man diese E rgebnisse auf die adjuvante Situation, könnte man durch den E insatz dieser Kombinationsprotokolle auf eine Verdoppelung des rezidivfreien Ü berlebens hoffen.
In mehreren laufenden Studien, z. B. der E ORTC in Europa und der CA LGB sowie der Intergroup in Nordamerika, wird diese Fragestellung aktuell untersucht. Die CA LGB-Studie setzt an 1260 Patienten mit einem komplett resezierten Kolonkarzinom des Stadiums III ( $\mathrm{T}_{\mathrm{x}} \mathrm{N}_{1-2} \mathrm{M}_{0}$ ) ohne chemotherapeutische Vorbehandlung eine adjuvante Therapie mit 5-FU/FA mit oder ohne I rinotecan ein [2]. Im Kontrollarm besteht die Therapie aus dem R oswell-Park-Schema mit 6 wöchentlichen Kurzinfusionen von Folinsäure und 5-Fluorouracil. D ie Zyklen werden alle 8 Wochen insgesamt 4-mal wiederholt (Behandlungsdauer 32 Wochen). Im experimentellen A rm werden pro Zyklus I rinotecan, Folinsäure und 5-Fluorouracil wöchentlich für 4 Wochen verabreicht, gefolgt von 2 Wochen Pause. Insgesamt werden 5 Zyklen dieser Kombinationstherapie verabreicht, so dass die Behandlungsdauer 30 Wochen beträgt. Primärer E ndpunkt dieser Studie ist das rezidivfreie Ü berleben, ein sekundärer $E$ ndpunkt das $G$ esamtüberleben. $E$ in wichtiges Ziel dieser Studie besteht auch darin, den E influss biologischer Prognosemarker auf das Ü berleben zu untersuchen. Zu diesem $Z$ weck wird das Primärtumorgewebe aller Patienten in einem zentralen Studienlabor systematisch untersucht. Dadurch sollen Subgruppen von Patienten identifiziert werden, die von der adjuvanten Therapie über- oder unterdurchschnittlich profitieren. Die E ORTC-Studie (darin eingegliedert die A ventisStudie 307) bezieht 1900 Patienten des Stadiums III (in der A ventis-Studie auch Patienten des Stadiums II) ein. Die Behandlung besteht aus 5-FU/FA nach dem A rdalan- oder de-G ramont-Schema, jeweils mit oder ohne Irinotecan. Die Therapiedauer beträgt jeweils ein halbes Jahr.

\section{Adjuvante Therapie im Stadium II}

$\mathrm{N}$ ach wie vor umstritten ist der Wert einer adjuvanten Chemotherapie im Stadium II (D ukes B). In einer kürzlich publizierten $M$ etaanalyse wurden die $D$ aten von 4006 Patienten aus den 4 randomisierten Studien C-01 bis C-04 des N SA B P (N ational Surgical A djuvant Breast and Bowel Project) unter diesem G esichtspunkt ausgewertet [3]. 41\% der behandelten Patienten hatten sich im Stadium D ukes B befunden. E s zeigte sich, dass in diesen Studien die Dukes-B-Patienten mindestens in

\begin{tabular}{ll}
\hline KARGER & ○ 2000 S. Karger G mbH , Freiburg \\
Fax +49 761 4520714 & A ccessible online at: \\
$\begin{array}{l}\text { E-mail Information@K arger.de } \\
\text { www.karger.com }\end{array}$ & www.karger.com/journals/onk
\end{tabular}

Prof.Dr. H.J.Schmoll M artin-L uther-U niversität

Klinik und Poliklinik für Innere M edizin

Ernst-G rube-Straße 40

D $-06120 \mathrm{H}$ alle (G ermany) 
gleichem $M$ aße von der Behandlung im jeweils wirksameren Studienarm profitierten wie die D ukes-C -Patienten.

Der U mstand, dass in vielen anderen Studien im Stadium II kein signifikanter $\mathrm{E}$ ffekt der adjuvanten Therapie nachweisbar war, ist wahrscheinlich auf die fehlende Risikostratifizierung der Patienten zurückzuführen. So konnten Jen et al. [4] bereits im Jahre 1994 aufzeigen, dass der A llelverlust des Chromosoms $18 \mathrm{q}$ bei kolorektalen Stadium-II-Tumoren mit einer dramatisch schlechteren Prognose verbunden ist (5-Jahres-Ü berlebensrate $54 \%$ gegenüber $93 \%$ bei Patienten ohne Allelverlust). Seither wurden von mehreren A rbeitsgruppen weitere einflussreiche molekulare Prognosemarker entdeckt, die mit einem mehrfach erhöhten R ezidiv- und Mortalitätsrisiko einhergehen.

Somit steht fest, dass das klinische Stadium nur eine grobe E inschätzung der individuellen Prognose ermöglicht. U m im $\mathrm{R}$ ahmen klinischer Studien den Nutzen einer möglicherweise marginal wirksamen adjuvanten Chemotherapie im Stadium II nachweisen zu können, wird daher eine sorgfältige Stratifizierung der Patienten anhand eines umfassenden molekulargenetischen Prognose-R atings erforderlich sein. In der laufenden CA LGB-Studie wird diesen neuen Erkenntnissen R echnung getragen. N eben potenziellen E influssfaktoren von Seiten der Chemotherapie (D osisintensität und Schema von 5-Fluorouracil, Expression von Thymidylat-Synthase und Dihydropyrimidindehydrogenase, funktioneller Status des A poptoseapparats, Expression von $\mathrm{M}$ ismatch-R eparaturgenen) werden auch zahlreiche biologische Malignitätsfaktoren bestimmt. Von dieser Studie erhofft man sich daher wertvolle A ufschlüsse auch für die klinische Praxis.

\section{Neoadjuvante Therapie beim Rektumkarzinom}

B eim lokal fortgeschrittenen R ektumkarzinom wurden kürzlich interessante Ergebnisse einer Phase-I-Studie mit einem multimodalen neoadjuvanten Therapiekonzept vorgestellt [5]. Die Patienten mit einem Rezidiv oder Primärtumor des Stadiums T3/4 erhielten simultan zu einer Strahlentherapie eine Chemotherapie mit 4 wöchentlichen Dosen I rinotecan und wöchentlichen Langzeitinfusionen von 5-Fluorouracil (über jeweils 5 Tage). Bei 20 bislang operierten Patienten wurde in $5 \mathrm{Fällen}$ eine pathologisch komplette R emission und in weiteren 6 Fällen ein minimaler R esttumor festgestellt. D ieses Ergebnis deutet darauf hin, dass man auch in dieser Therapiesituation durch die Kombination von Irinotecan mit den konventionellen Therapieverfahren einen Fortschritt erzielen kann, indem man die Operabilität erhöht und die R ezidivrate senkt.

\section{Adjuvante und neoadjuvante Therapie bei Lebermetastasen}

E in weiteres mögliches E insatzgebiet für neue Substanzen wie I rinotecan ist die adjuvante $C$ hemotherapie nach chirurgischer R esektion solitärer $M$ etastasen beim fortgeschrittenen Kolorektalkarzinom. In einer Gemeinschaftsstudie von EORTC, NCIC und GIVIO erhalten die Patienten im Standardarm wie bisher üblich erst bei einsetzender Progression eine B ehandlung mit 5-FU/FA. Im experimentellen A rm wird leider nur 5-FU/FA als adjuvante Therapie verabreicht. Hier wäre es wahrscheinlich sinnvoller gewesen, die derzeit wirksamste Kombinationstherapie, nämlich I rinotecan plus 5-FU/FA, einzusetzen, um den Behandlungseffekt zu maximieren.

$D$ ass ein intensiviertes Therapiekonzept in dieser Situation sinnvoll ist, zeigten die E rgebnisse einer weiteren adjuvanten Therapiestudie, in der die Patienten nach R esektion von kolorektalen Lebermetasen entweder eine systemische Chemotherapie mit 6Zyklen 5-F luorouracil (mit oder ohne Folinsäure) oder zusätzlich 6-mal eine intraarterielle Chemotherapie mit FUDR erhielten [6]. Nach 2 Jahren lag die lokalrezidivfreie Ü berlebensrate nach systemischer Chemotherapie bei $60 \%$ und nach systemischer + lokaler Chemotherapie bei $90 \%$ $(p<0,001)$. D a die systemische $M$ etastasierung durch die lokale Therapie nicht beeinflusst wurde, war der U nterschied in der 2-Jahres-G esamtüberlebensrate geringer ( 72 vs. $86 \%$ ), aber immer noch signifikant $(p=0,03)$. In der $\mathrm{N}$ achfolgestudie wird diese A rbeitsgruppe auch die systemische Komponente dieses postoperativen Therapieregimes intensivieren, indem sie I rinotecan mit hinzunimmt.

E ine andere, noch konsequentere Strategie wird in einer bald anlaufenden E ORTC-Studie eingeschlagen, indem eine intensive Chemotherapie bereits vor R esektion der L ebermetastasen, also in der neoadjuvanten Situation, verabreicht wird. D ies steht im E inklang mit der klinischen E rfahrung, dass sich kolorektale Lebermetastasen unter einer Chemotherapie oft gut zurückbilden und häufig operabel werden, so dass sich die Prognose oft deutlich verbessert. Damit kann der rationale E insatz neuer aktiver Substanzen in dieser Krankheitssituation dazu beitragen, das ursprünglich palliative Therapiekonzept in einen potenziell kurativen A nsatz zu verwandeln.

\section{Literatur}

1 Zalcberg J R, Siderov J, Simes J : The role of 5-fluorouracil dose in the adjuvant therapy of colorectal cancer. A nn O ncol 1996;7:42-46.

2 http://cancernet.nci.nih.gov/pdqfull.html\#clinical_trial $3 \mathrm{M}$ amounas $\mathrm{E}$, Wieand $\mathrm{S}$, Wolmark $\mathrm{N}, \mathrm{Bear}^{-} \mathrm{HD}$, A tkins J $\mathrm{N}$, Song $\mathrm{K}$, J ones J, R ockette $\mathrm{H}$ : Comparative efficacy of adjuvant chemotherapy in patients with D ukes' B versus D ukes' $C$ colon cancer: $R$ esults from four National Surgical A djuvant Breast and Bowel
Project adjuvant studies (C-01, C-02, C-03, and C-04) J Clin Oncol 1999;17:1349-1355.

4Jen J, Kim H, Piantadosi S, Liu ZF, Levitt RC, Sistonen $P, K$ inzler $K W$, Vogelstein $B, H$ amilton $S R$ : Allelic loss of chromosome $18 \mathrm{q}$ and prognosis in colorectal cancer. N Engl J M ed 1994;331:213-221.

5 Mitchell E, A hmad N, Fry R, A nne PR, Rakinic J, Goldstein S, R ose $L$, K aufmann A, H ightower $M$ Palazzo J, Boman B, Bonanni R, H oey D, Curran J Combined modality therapy of locally advanced or recurrent adenocarcinoma of the rectum: Preliminary report of a phase I trial of chemotherapy (CT) with CPT-11, 5-FU and concomitant irradiation (RT). Proc A m Soc Clin Oncol 1999;18:948(abstr).

6 Kemeny $N, H$ uang $Y$, Cohen A M, Shi W, Conti JA, B rennan M F, B ertino JR, Turnbull A D, Sullivan $D$, Stockman J, B lumgart LH, Fong Y : H epatic arterial infusion of chemotherapy after resection of hepatic metastases from colorectal cancer. N Engl J Med 1999;341:2039-2048. 